\title{
Where Grounding and Causation Part Ways: Comments on Jonathan Schaffer
}

\author{
Kathrin Koslicki \\ University of Alberta
}

\section{The Unity Hypothesis}

Metaphysicians have recently found it useful to apply the notion of ground to a wide range of phenomena. Jonathan Schaffer, for example, cites the following cases, among others, as exhibiting genuine grounding connections: ${ }^{1}$ the relation between true propositions and their truthmakers; the relation between higher-order (e.g., biological) and lower (e.g., physical) properties; the relation between complex truths (e.g., conjunctions) and simpler truths (e.g., their conjuncts); the relation between substances and their qualities or modes (e.g., an apple and its redness); as well as the relation between a non-empty set and its members. When presented with such an apparently heterogeneous collection of cases, however, one may legitimately wonder whether much of anything has been accomplished by subsuming these phenomena, and perhaps others as well, under a single general rubric of grounding. In response to this concern, it is not uncommon for proponents of grounding to respond that, by collecting the data in question together in this way, we have in fact gained a valuable insight, namely that a collection of correlations which might at first strike us as quite disparate in reality presents us with a unified phenomenon ("the Unity Hypothesis"). ${ }^{2}$

But does the notion of ground, as it has been recently employed by metaphysicians, in

${ }^{1}$ In what follows, when I refer to Jonathan Schaffer's work, I have in mind "Grounding in the Image of Causation" (this volume), unless otherwise noted.

${ }^{2}$ For an endorsement of the Unity Hypothesis, see for example Audi 2012, p. 689; Rosen 2010, p. 114; Schaffer 2009, pp. 376-377. 
fact point to a single unified phenomenon? I have argued (cf., Koslicki 2014) that the Unity

Hypothesis is open to several interpretations of varying strengths. In its strongest form (the "single-species" interpretation), the Unity Hypothesis states that there is only a single specific grounding relation and it is exemplified in all cases which allegedly present us with grounding connections. A somewhat weaker version of the Unity Hypothesis (the "single-genus" interpretation) allows for distinct specific grounding relations, but posits that these distinct specific grounding relations fall under a single generic kind, viz., grounding. A yet weaker reading of the Unity Hypothesis (the "mere resemblance" interpretation) requires only that the distinct relations which go under the name, "grounding", exhibit various objective similarities. ${ }^{3}$

Schaffer holds that the phenomenon of grounding exhibits the unity characteristic of a single genus. Given the many fairly obvious differences between the cases he classifies as exhibiting genuine grounding connections, however, the question arises as to what Schaffer's

${ }^{3}$ An interesting fourth option, which is well worth exploring further, is identified in Cameron forthcoming. Cameron challenges grounding theorists to consider the possibility that alleged grounding connections might exhibit a kind of unity that is weaker than that associated with the single-species or single-genus interpretation, but stronger than that associated with the mere-resemblance interpretation. The kind of unity in question, which Cameron brings to our attention, is that familiar from the Aristotelian notion of "pros hen" (or core-dependent) homonymy. As is well-known, when Aristotle says of certain central philosophical notions (e.g., being) that they are said in many ways, he often takes the notion in question to apply in a primary way to a certain central case (viz., substance) on which all the other applications of the notion are in some way dependent. Cameron issues the following challenge to grounding theorists. If a successful case cannot be made that alleged grounding connections fall under a single species or a single genus, and grounding theorists wish to avoid the charge of mere equivocation, then Aristotelian core-dependent homonymy ought to be considered as a possible fourth route towards a successful defense of the Unity Hypothesis. In that case, however, additional work is required of grounding theorists, since they must then establish that the notion of ground applies in a primary way to a certain central case on which all other alleged applications of the notion of ground are in some way dependent. To my knowledge, no extant account of grounding has availed itself, or is even particularly conducive to, the idea that ground is a core-dependent notion. 
evidence is for thinking that his notion of ground in fact picks out a single genus, rather than a looser assembly tied together merely by the presence of objective similarities. In his earlier work on the topic (especially Schaffer 2009), Schaffer's conception of grounding was not sufficiently fine-grained to differentiate his intended generic grounding relation from other well-founded partial orderings, such as the relation, is-a-substring-of, when applied to a domain whose minimal elements consist of the letters belonging to some alphabet. However, Schaffer now proposes to strengthen his case in favor of the generic unity of grounding by taking seriously the analogy between non-metaphysical causation and grounding. ${ }^{4}$ (In what follows, I refer to nonmetaphysical causation simply as "causation".) More specifically, Schaffer argues that (i) grounding and causation are similar with respect to their content, internal structure and external connections to surrounding concepts; that (ii) both grounding and causation are best approached through a single formalism, viz., that utilized by structural equation models of causation; and that (iii) both grounding and causation belong to a small elite group of dependence relations which have the power to back explanation. As a result, if Schaffer's more recent arguments are successful, the hypothesis that grounding presents us with a unified phenomenon has at least as much plausibility as the analogous hypothesis in the case of causation. ${ }^{5}$

${ }^{4}$ I speak here of non-metaphysical causation, since some proponents of grounding, including Schaffer himself, have taken grounding to be a kind of causation, viz., metaphysical causation. I therefore employ the phrase, "non-metaphysical causation", to pick out a causal determination relation which proponents of grounding would want to distinguish from whatever determination relation(s) these theorists intend to single out when they speak of grounding. For a very different construal of metaphysical causation, from within Aristotle's framework, see Koslicki forthcoming.

${ }^{5}$ Schaffer takes for granted, in his discussion, that the Unity Hypothesis is plausible for the case of causation. I will not challenge this claim, since my focus is on Schaffer's analogy between grounding and causation. Thus, I am willing to grant Schaffer's assumptions 
In what follows, I will focus on the arguments provided by Schaffer in support of the second step needed to establish the intended analogy between causation and grounding, viz., that both notions are best approached through the lense of a single formalism, namely that utilized by structural equation models of causation. Below, I will present several concerns which suggest that the structural equation model does not transfer as smoothly from the case of causation to the case of grounding as Schaffer would have us believe. If it can in fact be shown that significant differences surface in how the formalism in question applies to the two types of phenomena in question, Schaffer's attempt at establishing an analogy between grounding and causation has thereby been weakened and, as a result, the application of the Unity Hypothesis to the case of grounding once again stands in need of justification. ${ }^{6}$

\section{Disanalogies between Grounding and Causation}

\section{II.1 Two Scenarios}

To bring out some of the central disanalogies between grounding and causation, I will first consider a simple example which Schaffer uses to illustrate how he intends to apply the

concerning causation, for the sake of the argument, and will call into question, in what follows, only Schaffer's claim that grounding and causation in fact behave analogously in relevant respects. However, another possible route towards weakening Schaffer's case in favor of the alleged generic unity of grounding would be to object to his assumption that causation exhibits at least the degree of unity indicative of the presence of a single genus.

${ }^{6}$ For further discussion of the application of the Unity Hypothesis to the case of grounding, see also Wilson (2014), especially Section V, "Is Grounding Needed as a General Unifier of the Specific Grounding Relations?", pp. 567-575. Wilson considers (and rejects) the idea that the analogy between causation and grounding might serve as a possible route towards defending the unity of grounding (cf., Section V.iii, "Contrasting Grounding and Causation as Potential Unifiers", p. 575). 
structural equation model to the case of causation. ${ }^{7}$ In the scenario in question ("Scenario 1"), we are to assume that a rock's being thrown against a window is causally related to a window's shattering. To represent this scenario using the structural equation model of causation, we define first a signature, $\mathrm{S} 1=<\mathrm{U} 1, \mathrm{~V} 1, \mathrm{R} 1>$, where $\mathrm{U} 1$ is a finite set of exogenous variables representing the independent (or initial) conditions, $\mathrm{V} 1$ is a finite set of endogenous variables representing the dependent conditions, and R1 is a mapping function which maps every variable in $\mathrm{U} 1 \cup \mathrm{V} 1$ to either 0 or 1 . In the scenario in question, $\mathrm{U} 1$ can be thought of as containing the variable, Thrown, which represents the event of the rock's being thrown against the window (as contrasting with the rock's not being thrown against the window); and V1 can be thought of as containing the variable, Shatter, which represents the event of the window's shattering (as contrasting with the window's not shattering). R1 maps Thrown to 1, if the event of the rock's being thrown against the window occurs; to 0 otherwise. Likewise, R1 maps Shatter to 1, if the event of the window's shattering occurs; to 0 otherwise. Secondly, to represent how the endogenous variable, Shatter, is to be evaluated as a result of the value assigned by R1 to the exogenous variable, Thrown, we define a linkage, $\mathrm{L} 1=<\mathrm{S} 1, \mathrm{E} 1>$, where $\mathrm{S} 1$ is the signature just specified and E1 is a set of structural equations which specifies which value is assigned to Shatter on the basis of the value assigned to Thrown. In this case, E1 assigns the value 1 to

\footnotetext{
${ }^{7}$ Again, in line with my remarks in the previous note, I will, for the purposes of this discussion, grant Schaffer's assumption that the structural equation model does in fact yield a successful framework by means of which to approach causation, since I am currently concerned only with Schaffer's attempt at establishing an analogy between grounding and causation by way of the thesis that the structural equation model applies equally well to both cases. A further strategy by which Schaffer's proposed analogy between grounding and causation could be disputed would be to cast doubt on the successes he claims for structural equation approaches to causation.
} 
Shatter, if Thrown is assigned 1; and E1 assigns the value 0 to Shatter, if Thrown is assigned 0.

Thirdly, to represent what actually happens in the scenario in question, we define an assignment, $\mathrm{M} 1=<\mathrm{L} 1, \mathrm{~A} 1>$, where $\mathrm{L} 1$ is the linkage just specified and $\mathrm{A} 1$ is a function mapping Thrown to either 0 or 1 , depending on whether the rock is in fact thrown. In the event that the rock is thrown, the value assigned to Shatter in the model in question is set to 1 as well.

Next, consider a second simple example Schaffer employs to illustrate how he takes the structural equation model to apply to an alleged case of grounding. In this scenario ("Scenario 2"), we are to assume that a shirt's being red (as opposed to blue) is grounded in the shirt's being maroon (as opposed to navy). We again define first a signature, $\mathrm{S} 2=<\mathrm{U} 2, \mathrm{~V} 2, \mathrm{R} 2>$, where $\mathrm{U} 2$ is a set Schaffer labels " $\{$ Determinate $\}$ ", V2 is a set Schaffer labels " $\{$ Determinable $\}$ ", and R2 is the following mapping function: R2 maps Determinate to 1 if the shirt is maroon and to 0 if the shirt is navy; and R2 maps Determinable to 1 if the shirt is red and to 0 if the shirt is blue. In alleged cases of grounding, we are to think of the exogenous variable in $\mathrm{U} 2$ as specifying the "fundamental" conditions (as opposed to the initial conditions, in the case of causation). ${ }^{8}$ Secondly, we define a linkage, $\mathrm{L} 2=<\mathrm{S} 2, \mathrm{E} 2>$, where $\mathrm{S} 2$ is the signature just specified and $\mathrm{E} 2$ is a set of structural equations which specifies which value is to be assigned to the endogenous

${ }^{8}$ When Schaffer proposes to read the exogenous variables, in the alleged grounding scenario at hand, as representing the "fundamental" conditions (as opposed to the initial conditions, in the causal case), we must read him as having in mind relative, rather than absolute, fundamentality. Grounding theorists tend not to think of such states of affairs as the shirt's being maroon as absolutely fundamental, i.e., as a state of affairs which does not obtain in virtue of some other more fundamental state of affairs' obtaining. Thus, in Scenario 2, the exogenous variables should be understood as representing conditions which, relative to the scenario at hand, are assumed to be more fundamental than the dependent conditions represented by the endogenous variables; but they need not stand for conditions which are taken to be fundamental absolutely. 
variable, Determinable, on the basis of the value assigned to the exogenous variable,

Determinate. In this case, E2 assigns 1 to Determinable if 1 is assigned to Determinate; and 0 to Determinable if 0 is assigned to Determinate. Thirdly, to represent what actually obtains in the scenario in question, we define an assignment, $\mathrm{M} 2=<\mathrm{L} 2, \mathrm{~A} 2>$, where $\mathrm{L} 2$ is the linkage just specified and A2 is a function mapping Determinate to 0 or 1 depending on whether the shirt is in fact maroon or navy. In the event that the shirt is maroon, the value assigned to Determinable will be set to 1 as well in the model under consideration. This representation, in Schaffer's words, encodes "how the shirt's determinate shade sets its determinable color" (Schaffer 2015, Section 3.1, p. 16).

\section{II.2 Evaluating the Variables}

In Scenario 1, the exogenous and endogenous variables can be understood to range over events (e.g., the rock's being thrown against the window and the window's shattering) which we can conceive of as either occurring or not occurring. What are the values over which the exogenous and endogenous variables range in Scenario 2? In what follows, I will interpret the variables in Scenario 2 as ranging over states of affairs (e.g., the shirt's being maroon and the shirt's being red) which we can conceive of as either obtaining or not obtaining. Given Schaffer's conception of grounding as a worldly relation which can back (but is not to be identified with) explanation, it is important here to construe the relata of grounding (e.g., states of affairs) equally as worldly entities with no admixture of linguistic, conceptual or otherwise representational content. 


\section{II.3 Determinate/Determinable Grounding and Massive Causal Preemption}

In Scenario 1, we are to think of the values assigned to the variables, Thrown and Shatter, as events (viz., the rock's being thrown against the window and the window's shattering), which can either occur or not occur. If the first event consisting in the rock's being thrown against the window in fact occurs, then (so we are to assume) the second event consisting in the window's shattering occurs as well. Given the interpretation proposed in II.2, we will think of the values assigned to the variables in Scenario 2, Determinate and Determinable, as states of affairs (viz., the shirt's being maroon and the shirt's being red), which can either obtain or not obtain.

But now the following disanalogy between Scenario 1 and Scenario 2 surfaces, as these scenarios are represented by the above models. In Scenario 1, the contrast between the rock's being thrown against the window and the rock's not being thrown against the window corresponds to the values, 1 and 0 , assigned to the exogenous variable, Thrown. (And the same holds for Shatter.) In contrast, in Scenario 2, the exogenous variable, Determinate, is to be assigned the value 1 if the shirt is maroon, and the value 0 if the shirt is navy. But the shirt's being navy is itself a distinct state of affairs, numerically different from the state of affairs which consists in the shirt's being maroon. Both states of affairs (viz., the shirt's being maroon and the shirt's being navy) can be conceptualized as either obtaining or not obtaining. Moreover, there is no direct link between the non-obtaining of one of these states of affairs and the obtaining of the other: for if the shirt fails to be maroon, it is not thereby required to be navy instead, since all other determinate shades of color are options for the shirt as well. (Similar remarks apply to the evaluation of the endogenous variable, Determinable, as well.)

Given the aptness constraints Schaffer imposes on his models (cf., Section 2.4 for the 
causal case and Section 3.3 for the case of grounding), the model representing Scenario 2 would need to include distinct variables for the various states of affairs in question: e.g., an exogenous variable, Maroon, representing the state of affairs of the shirt's being maroon; an exogenous variable, Navy, representing the state of affairs of the shirt's being navy; an endogenous variable, Red, representing the state of affairs of the shirt's being red; and an endogenous variable, Blue, representing the state of affairs of the shirt's being blue. The contrasting values, 0 and 1 , which can be assigned to these variables, in each case, would correspond to each state of affairs in question either obtaining or not obtaining.

In order to preserve the analogy between causation and grounding, then, Scenario 2 would need to be modeled as follows. We define first the signature, $\mathrm{S} 2 *=<\mathrm{U} 2 *, \mathrm{~V} 2 *, \mathrm{R} 2 *>$, where $\mathrm{U} 2 *$ contains the exogenous variable, Maroon, V2* contains the endogenous variable, Red, and R2* is the following mapping function: $\mathrm{R} 2 *$ assigns 1 to Maroon if the shirt is maroon and 0 otherwise; and R2* assigns 1 to Red if the shirt is red and 0 otherwise. Secondly, we define a linkage, $\mathrm{L} 2 *=<\mathrm{S} 2 *, \mathrm{E} 2 *>$, where $\mathrm{S} 2 *$ is the signature just specified and $\mathrm{E} 2 *$ is a set of structural equations which specifies which value is to be assigned to the endogenous variable, Red, on the basis of the value assigned to the exogenous variable, Maroon. In this case, E2* assigns 1 to Red if 1 is assigned to Maroon; and 0 to Red if 0 is assigned to Maroon. Thirdly, to represent what actually obtains in the scenario in question, we define an assignment, $\mathrm{M} 2 *=<\mathrm{L} 2 *, \mathrm{~A} 2 *>$, where L2* is the linkage just specified and A2* is a function mapping Maroon to 0 or 1 depending on whether the shirt is in fact maroon. In the event that the shirt is maroon, the value assigned to Red will be set to 1 as well in the model under consideration.

The current representation of Scenario 2 differs from the earlier one in the following two 
main respects. First, the contrast class represented by the values, 0 and 1 , is now to be understood as corresponding to one and the same state of affairs (e.g., the shirt's being maroon) either obtaining or not obtaining. Secondly, an assignment which sets Maroon to 0 (denoting that the state of affairs consisting in the shirt's being maroon does not obtain) must be assumed to be compatible with a whole range of alternatives, all of which are represented by means of distinct variables in the model in question, e.g., Crimson (representing the state of affairs consisting in the shirt's being crimson), Navy (representing the state of affairs consisting in the shirt's being navy), and so on.

There is now reason to doubt whether, given this second representation of Scenario 2, the model at hand actually encodes "how the shirt's determinate shade sets its determinable color", as Schaffer claims in the above cited remark. Given that Maroon's being set to 0 leaves open, for example, whether Crimson should be set to 1 in the scenario in question, E2* would in some cases generate the wrong results. After all, there are many different ways in which the shirt can be red, and the state of affairs represented by Maroon captures only one of these ways. It would therefore be incorrect to define E2* in such a way that it assigns 0 to Red whenever 0 is assigned to Maroon; for the scenario in question may be one in which the shirt is nevertheless red, only in some other way, e.g., by being crimson rather than maroon. This result presents a counterexample to Schaffer's slogan, "wiggle the ground, and the grounded wiggles" (Schaffer 2015, Section 3.2, p. 19): for in a case in which we "wiggle the ground" by imagining the shirt's color to be changed from maroon to crimson, say, it is not the case that thereby "the grounded wiggles" as well, since the shirt continues to be red, only in a different way.

In addition, a further general feature of the determinate/determinable link is not currently 
represented in the model in question. Unlike in the causal case, it is metaphysically necessary that the shirt always has some determinate and some corresponding determinable shade of color, regardless of which determinate shade of color the shirt in fact has. ${ }^{9}$ Thus, if Schaffer's endogenous variable, Determinable, in Scenario 2 in fact did represent the state of affairs consisting in the shirt's exemplifying some determinable shade of color or other, its value would always have to be set to 1 (assuming that we are representing what is metaphysically possible), regardless of what value is assigned to the exogenous variable in question.

There are thus two main deficiencies in how the determinate/determinable link is currently represented in the revised model just specified: first, the model fails to capture adequately the specific dependence relation which obtains between a particular determinate/determinable pair (e.g., the shirt's being maroon and the shirt's being red); and, secondly, the model does not speak to the general dependence relation which obtains between determinables and determinates of a certain kind (e.g., all determinate shades of color and their corresponding determinables in general).

When the structural equation model is applied to an alleged case of determinable/determinate grounding, the grounding scenario in question is in fact more aptly compared to a causal scenario involving massive causal preemption, i.e., a scenario in which a single effect can be brought about by multiple alternative causes, each of which is individually sufficient to bring about the effect in question and each of which occurs only if none of the others

${ }^{9}$ In contrast, in the causal case, we are dealing with contingently occurring events, viz., the rock's being thrown against the window and the window's shattering. Even the causal connection which obtains between the rock's being thrown against the window and the window's shattering is not metaphysically necessary, though it is perhaps governed by some other form of necessity, e.g., nomological necessity. 
occur. As it stands, it is not clear, even in the causal case, how the structural equation model, as described by Schaffer, would produce the correct results in a case of massive causal preemption. At most, then, we are dealing with a situation in which a supposedly clear case of grounding is comparable to a problematic case of causation, one which has led to headaches for extant theories of causation including, by Schaffer's own admission, the structural equation model of causation.

When Scenario 2 is represented in the revised way presented above, we notice furthermore that, in an alleged case of determinate/determinable grounding, the "fundamental" conditions represented by the exogenous variables are not truly independent of the so-called “dependent" conditions represented by the endogenous variables. Rather, each determinable shade of color (e.g., red) sets its own range of alternative possibilities, consisting of the corresponding determinate shades of color (e.g., maroon or crimson or ...). The shirt can only exhibit a certain determinable shade of color (e.g., red) by exhibiting one of the admissible determinate shades of color (e.g., maroon or crimson or ...) which falls into the range of alternatives set by the relevant determinable shade.

In contrast to the causal case, there are thus dependence relations running in both directions in an alleged case of determinate/determinable grounding: a rigid dependence relation connecting particular states of affairs, running from the shirt's exemplifying some determinate shade of color (e.g., maroon) to its exemplifying the corresponding determinable shade of color (viz., red); but also a generic dependence relation connecting the shirt's exemplifying some determinable shade of color (e.g., red) to the shirt's exemplifying some one particular determinate shade of color (e.g., maroon) which falls into the relevant range of admissible 
alternatives (viz., maroon or crimson or ...) set by the determinable shade in question. If we take the variable, Color, to represent the state of affairs consisting in the shirt's exemplifying some determinable shade of color or other, then the laws governing metaphysical possibility (if there are such laws) dictate that the value assigned to Color must always be 1 (assuming that colorless shirts are metaphysically impossible). Given that it is metaphysically necessary that the value assigned to Color is always 1, it is also metaphysically necessary that one of the variables, Red, Blue, etc., be set to 1 as well. Whichever one of Red, Blue, etc. is set to 1 , it then furthermore follows with metaphysical necessity from this assignment that one of the variables representing the more specific admissible possibilities falling into the range of alternatives in question, e.g., Crimson, Maroon, etc., is also set to 1, though it is not metaphysically necessary for any one in particular, e.g., Crimson, to be set to 1. In order for a model to capture the general determinate/determinable link, the following two-way dependencies would need to be incorporated in some fashion into the representation of the system in question as well:

(a) Overall Generic Determinable Outcome:

It is metaphysically necessary that Color is set to 1 .

(b) Generic Downward Dependence (from endogenous to endogenous variables): It is metaphysically necessary that one of Red or Blue or ... is set to 1 .

(c) Generic Downward Dependence (from endogenous to exogenous variables): It is metaphysically necessary that, if Red is set to 1, then one of Maroon or Crimson or ... is set to 1 as well.

(d) Rigid Upward Dependence (from exogenous to endogenous variables): It is metaphysically necessary that, if Maroon is set to 1, then Red and Color are 
set to 1 as well.

The very fact that two-way dependencies, such as those illustrated in (b)-(d), are implied by any particular determinate/determinable connection calls into question the applicability of the structural equation model to this alleged case of grounding, since the aptness constraints imposed on the model require that dependence relations run in only one direction, viz., from the conditions represented by the exogenous variables to the conditions represented by the endogenous variables, and not the other way around. Given the presence of such two-way dependencies, the allegedly "fundamental" conditions are only recombinable with some restrictions, contrary to what is required by the "free recombination" principle Schaffer imposes on the exogenous variables (Schaffer 2015, Section 3.3, p. 21).

\section{II.4 The "Non-Identity" Constraint}

Finally, I want to consider the first aptness constraint Schaffer imposes on his models. In the case of causation (cf., Section 2.4), Schaffer's first aptness constraint (“Distinctness") requires that the values assigned to distinct variables must all correspond to distinct events in the system, where "distinctness" here is meant to rule out both numerical identity and mereological overlap between events. In the case of grounding (cf., Section 3.3), the first aptness constraint ("Non-Identity") requires only that the values of distinct variables correspond to "non-identical" entities in the system. "Non-identity" is meant to be a weaker condition than "distinctness", which is now defined as follows (Schaffer 2015, Section 3.3, p. 21):

An entity, $\mathrm{x}$, and an entity, $\mathrm{y}$, are "distinct" if and only if (i) $\mathrm{x}$ and $\mathrm{y}$ are numerically distinct; and (ii) $\mathrm{x}$ and $\mathrm{y}$ are not "connected by grounding”, i.e., it is not the case that (a) 
$\mathrm{x}$ grounds $\mathrm{y}$ or (b) $\mathrm{y}$ grounds $\mathrm{x}$ or (c) $\mathrm{x}$ and $\mathrm{y}$ have a common ground $\mathrm{z}$.

Entities, $\mathrm{x}$ and $\mathrm{y}$, may be "non-identical" without also being "distinct", e.g., if one of the grounding connections mentioned in (ii) holds between $\mathrm{x}$ and $\mathrm{y}$. For example, in the revised model proposed for Scenario 2 above, distinct variables (viz., Maroon and Red) are reserved for the state of affairs consisting in the shirt's being maroon and the state of affairs consisting in the shirt's being red, even though the two states of affairs in question do not satisfy the definition of "distinctness" just offered, since they are connected by grounding (viz., the shirt is said to be red in virtue of its being maroon).

Presumably, the earlier definition of "distinctness", operative in the causal case, as excluding both numerical identity and mereological overlap is no longer appropriate for the case of grounding, since some entities in a given system ought to be represented via distinct variables even though they overlap mereologically. For example, if states of affairs are conceived of as having merelogical constituents of some kind, then the states of affairs represented by Maroon and Red overlap, since they both have the shirt as a constituent.

But now the worry arises that the new formulation of the first aptness constraint creates a circularity which was not present in the application of the structural equation model to the case of causation..$^{10}$ Numerical identity and mereological overlap are not causal notions. Thus, when we are engaged in the evaluation of a causal scenario, reading "distinct" as neither numerically identical nor mereologically overlapping is not overtly question-begging. In contrast, reading "distinct" as neither numerically identical nor connected by grounding does appear to be

${ }^{10}$ The threat of circularity cited here arises even when we take into consideration Schaffer's remarks concerning the non-reductiveness of the structural equation approach to causation or grounding. 
problematic in the context of evaluating an alleged grounding connection. After all, according to Schaffer's advertisement, one of the benefits of applying the structural equation model to the case of causation is supposed to be that this approach can distinguish mere correlations from causal connections (viz., the availability of "discovery algorithms"). Thus, when confronted with a type of phenomenon, $\mathrm{x}$, and a type of phenomenon, $\mathrm{y}$, which are correlated (e.g., smoking and lung cancer), we may ask "Does x cause y?" and, ideally, the structural equation model of causation should generate the correct answer to this question. Similarly, given Schaffer's account, we should expect to be able to ask the analogous question in the case of grounding, "Does x ground y?", when confronted by a type of phenomenon, $\mathrm{x}$, and a type of phenomenon, $\mathrm{y}$, which are correlated in some way (e.g., maroonness and redness). Ideally, if Schaffer's analogy between causation and grounding is successful, we should be able to expect the structural equation model, when applied to an alleged case of grounding, to distinguish correctly between the presence of a mere correlation and the presence of a genuine grounding connection. Given this success condition, we should not be forced to specify at the outset whether $\mathrm{x}$ and $\mathrm{y}$ are connected via some grounding connection, just as we should not be forced, in the case of causation, to specify at the outset whether $\mathrm{x}$ and $\mathrm{y}$ are causally connected. If the initial set-up of the model does require us to determine at the outset whether $\mathrm{x}$ and $\mathrm{y}$ are causally connected or ground-theoretically connected, then the only open question left to be resolved by the application of the structural equations in question concerns the direction in which causal or grounding connection runs in the system at issue. I take it, however, that one of the main selling points of the structural equation approach to causation and grounding was also meant to be the ability of a 
particular model to detect the presence of a genuine causal or ground-theoretic connection. ${ }^{11}$

\section{Conclusion}

Given the foregoing remarks, we thus have reasons to believe that significant disanalogies exist between causation and grounding. In particular, I have attempted to show that the application of the structural equation model to both types of phenomena leads to very different results: whatever the merits of the structural equation approach to the case of causation, its application to the case of grounding is much less straightforward than Schaffer's attempted analogy between causation and grounding leads us to believe. I thus part ways with Schaffer's claim that, in applying the formalism utilized by structural equation models of causation to the case of grounding: "Mathematically nothing has changed, beyond the purely decorative matter of the labels on the variables" (Schaffer 2015, Section 3.1, p. 16). The application of a single formalism to both grounding and causation constitutes a crucial step in Schaffer's arguments in support of the unity of grounding. To the extent that this step can be blocked, the hypothesis that grounding exhibits the unity characteristic of a single genus is thereby once again called into question.

${ }^{11}$ The presence of two-way dependencies in the grounding case cited at the end of the previous section furthermore creates problems for a model's success in predicting even the direction of the alleged grounding connection present in a given case. 


\section{$\underline{\text { References }}$}

Audi, Paul (2012). Grounding: Toward a Theory of the In-Virtue-Of Relation. Journal of Philosophy, 109(12), 685-711.

Cameron, Margaret (forthcoming). Is Grounding Said-in-many-ways? Studia Philosophica Estonica. Special issue on ontological priority and essence in Aristotle and Aristotelian Metaphysics, edited by Riin Sirkel and Tuomas Tahko.

Chalmers, David, Manley, David, and Wasserman, Ryan (eds.) (2009). Metametaphysics: New Essays on the Foundations of Ontology. Oxford: Clarendon Press.

Hale, Bob, and Hoffman, Aviv (Eds.) (2010). Modality: Metaphysics, Logic, and Epistemology, Oxford: Oxford University Press.

Koslicki, Kathrin (2014). The Coarse-Grainedness of Grounding. Oxford Studies in Metaphysics, 306-349.

Koslicki, Kathrin (forthcoming). The Causal Priority of Form. Studia Philosophica Estonica. Special issue on ontological priority and essence in Aristotle and Aristotelian Metaphysics, edited by Riin Sirkel and Tuomas Tahko.

Rosen, Gideon (2010). Metaphysical Dependence: Grounding and Reduction. In B. Hale and A. Hoffman (Eds.), 109-136.

Schaffer, Jonathan (2009). On What Grounds What. In D. Chalmers, D. Manley and R. Wasserman (Eds.), 347-383.

Schaffer, Jonathan (2015). Grounding in the Image of Causation. This volume.

Wilson, Jessica (2014). No Work for a Theory of Grounding. Inquiry, 57(5-6), 535-579. 